\title{
Classification of barley U-box E3 ligases and their expression patterns in response to drought and pathogen stresses
}

\author{
Moon Young Ryu ${ }^{2,3+}$, Seok Keun Cho ${ }^{2,3+}$, Yourae Hong ${ }^{4}$, Jinho Kim ${ }^{4}$, Jong Hum Kim²,3, Gu Min Kim²,3, \\ Yan-Jun Chen ${ }^{1}$, Eva Knoch ${ }^{1}$, Birger Lindberg Møller ${ }^{1}$, Woo Taek Kim², ${ }^{2,3^{*}}$, Michael Foged Lyngkjær ${ }^{1 *}$ and \\ Seong Wook Yang ${ }^{1,2,3^{*}}$
}

\begin{abstract}
Background: Controlled turnover of proteins as mediated by the ubiquitin proteasome system (UPS) is an important element in plant defense against environmental and pathogen stresses. E3 ligases play a central role in subjecting proteins to hydrolysis by the UPS. Recently, it has been demonstrated that a specific class of E3 ligases termed the U-box ligases are directly associated with the defense mechanisms against abiotic and biotic stresses in several plants. However, no studies on U-box E3 ligases have been performed in one of the important staple crops, barley.

Results: In this study, we identified 67 putative U-box E3 ligases from the barley genome and expressed sequence tags (ESTs). Similar to Arabidopsis and rice U-box E3 ligases, most of barley U-box E3 ligases possess evolutionary wellconserved domain organizations. Based on the domain compositions and arrangements, the barley U-box proteins were classified into eight different classes. Along with this new classification, we refined the previously reported classifications of U-box E3 ligase genes in Arabidopsis and rice. Furthermore, we investigated the expression profile of $67 \mathrm{U}$-box E3 ligase genes in response to drought stress and pathogen infection. We observed that many U-box E3 ligase genes were specifically up-and-down regulated by drought stress or by fungal infection, implying their possible roles of some U-box E3 ligase genes in the stress responses.

Conclusion: This study reports the classification of U-box E3 ligases in barley and their expression profiles against drought stress and pathogen infection. Therefore, the classification and expression profiling of barley U-box genes can be used as a platform to functionally define the stress-related E3 ligases in barley.
\end{abstract}

Keywords: Barley, Hordeum vulgare, Ubiquitin proteasome system (UPS), Biotic stress, Abiotic stress

\section{Background}

The ubiquitin proteasome system (UPS) orchestrates turnover of a large number of proteins in eukaryotic cells and thereby regulates cellular responses to external and internal stimuli while maintaining house-keeping functions $[1,2]$. The UPS is composed of three specific

\footnotetext{
*Correspondence: wtkim@yonsei.ac.kr; mlyn@plen.ku.dk; swyang@plen.ku.dk ${ }^{\dagger}$ Moon Young Ryu and Seok Keun Cho contributed equally to this work. ${ }^{2}$ Department of Systems Biology, College of Life Science and Biotechnology, Yonsei University, Seoul 120-749, Korea

'Plant Biochemistry Laboratory, Department of Plant and Environmental Sciences, University of Copenhagen, Thorvaldsensvej 40, DK-1871 Frederiksberg C, Copenhagen, Denmark

Full list of author information is available at the end of the article
}

enzyme-types 1) ubiquitin-activating E1 enzymes, 2) ubiquitin-conjugating E2 enzymes and 3) ubiquitin E3 ligase enzymes. Through multiple ubiquitination cycles, specific proteins are targeted to the proteasome for degradation [3-5]. In plants, the importance of the UPS system is exemplified in Arabidopsis, where about 6\% of the Arabidopsis genome or about 1600 genes encode core components of the UPS, including two E1 enzymes, at least 37 E2 enzymes and approximately 1.400 E3 ligases [1]. In general, the conjugation of ubiquitin(s) to a specific target protein is determined by the type of E3 ligase. The E3 ligases are classified into three families, the HECT-type, RING-type, and U-box-type E3 ligases,

(c) The Author(s). 2019 Open Access This article is distributed under the terms of the Creative Commons Attribution 4.0 International License (http://creativecommons.org/licenses/by/4.0/), which permits unrestricted use, distribution, and reproduction in any medium, provided you give appropriate credit to the original author(s) and the source, provide a link to the Creative Commons license, and indicate if changes were made. The Creative Commons Public Domain Dedication waiver (http://creativecommons.org/publicdomain/zero/1.0/) applies to the data made available in this article, unless otherwise stated. 
according to their functional domains. Among those, U-box-type E3 ligase is the smallest family with approximately 60 members in Arabidopsis [6-8]. The U-box containing proteins have been assigned as PLANT U-box (PUB) enzymes. All the defined PUB proteins in Arabidopsis and rice were named by consecutively numbering after the term PUB, except for the Arabidopsis U-box protein CHIP (Carboxyl terminus of HSC70interacting protein) [9]. Based on the sequence of 63 identified Arabidopsis AtPUB proteins and 77 rice OsPUB proteins, the proteins were assigned into nine different PUB classes according to their domain characteristics $[8,10]$ (Fig. 1c). Class I members (1 Arabidopsis; 1 rice) are homologues of the yeast UFD2 (ubiquitin fusion degradation protein 2) which contains a UFD2 domain, known to interacts with the AAA family ATPase CDC48 protein [12]. Class II members (29 Arabidopsis; 28 rice) possess a variable number of Armadillo repeats (ARM) in their $\mathrm{C}$-termini. These are thought to form an $\alpha$-solenoid structure that might constitute a protein interaction domain [12-14]. Class III members (12 Arabidopsis; 16 rice) are suggested to possess a GKL motif (a conserved Glycine (G), Lysine (K)/Arginine (R) residues and its leucine rich residues) located close to the C-terminus [10, 12]. Class IV members (16 Arabidopsis; 9 rice) possess a serine/threonine kinase domain at the $\mathrm{C}$-termini. Class $\mathrm{V}$ members (7 Arabidopsis; 8 rice) are characterized as PUB proteins without any additional recognizable domains [12]. Class VI members (2 Arabidopsis; 2 rice) possess a WD40 domain, which constitutes a well-known protein-to-protein interaction motif. Class VII members (1 Arabidopsis; 6 rice) contain a tetratrico-peptide repeat (TRP) domain, which has been shown to mediate protein-protein interactions [10]. Class VIII is rice specific and contains only one member, which in addition to the U-box domain possesses a TRP domain and a kinase domain. Class IX is Arabidopsis specific and contains two members with a MIF4G-type domain (Fig. 1b). The U-box E3 ligase family in grapevine [15] and Medicago [16] has been separated into

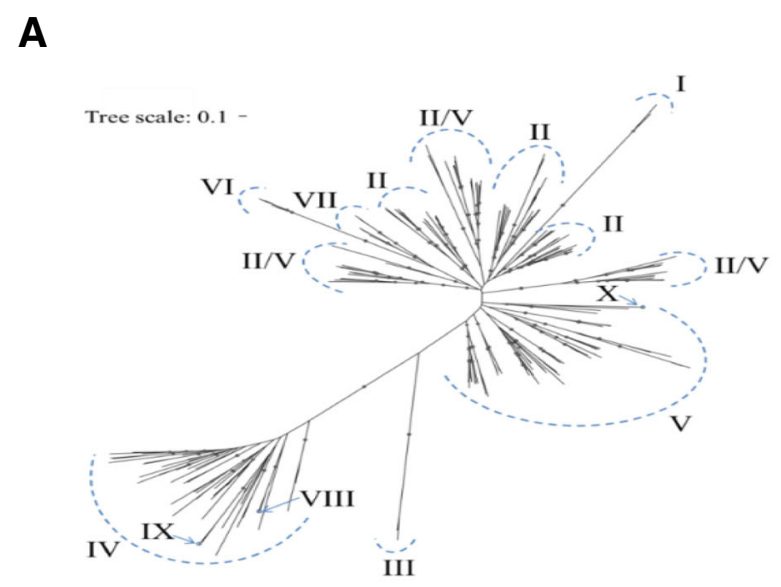

C
\begin{tabular}{|c|c|c|c|c|}
\hline \multirow{2}{*}{ Class } & \multicolumn{3}{|c|}{ No. of proteins } \\
\cline { 2 - 5 } & $\begin{array}{c}\text { HvPUB } \\
(67)\end{array}$ & $\begin{array}{c}\text { OsPPUB } \\
(77)\end{array}$ & $\begin{array}{c}\text { AtPUB } \\
(64)\end{array}$ \\
\hline \multicolumn{2}{|c|}{ Class I } & 1 & 1 & 1 \\
\hline \multirow{2}{*}{ Class II } & a & 19 & 19 & 17 \\
\cline { 2 - 5 } & b & 8 & 11 & 10 \\
\hline Class III & 1 & 1 & 1 \\
\hline Class IV & 11 & 17 & 14 \\
\hline Class V & 21 & 20 & 16 \\
\hline Class VI & 3 & 2 & 2 \\
\hline Class VII & 2 & 5 & 1 \\
\hline Class VIII & 0 & 1 & 0 \\
\hline Class IX & 0 & 0 & 2 \\
\hline Class X & 1 & 0 & 0 \\
\hline
\end{tabular}

B

Monocot

Dicot
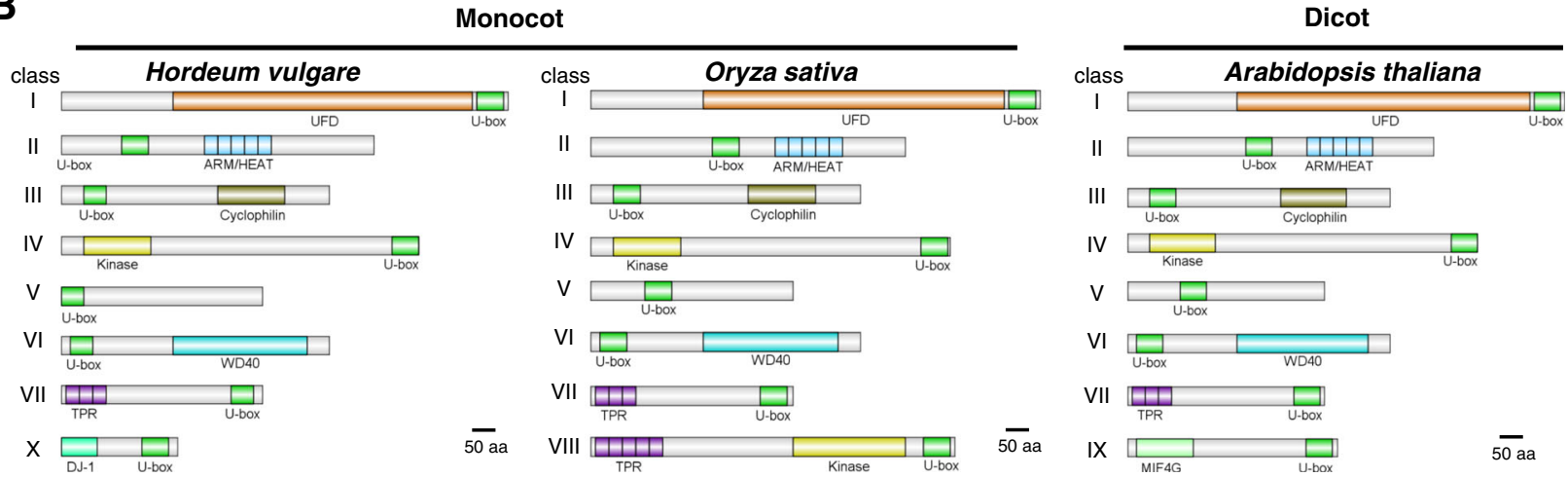

Fig. 1 Phylogenetic identification and domain structures of the 67 PUB genes in barley. a. Full-length amino-acid sequences of PUB genes in Arabidopsis, rice and barely were analyzed using the Clustal X2 software. The tree was constructed by neighbor-joining method after bootstrap analysis for 1000 replicates [1 1]. b. Domain structures of the 67 PUB genes into 8 different classes. Green box, U-box domain; brown box, UFD2 UB chain assembly domain; sky blue box, ARM repeat domain; yellow box, kinase domain; cyan box, WD40 protein interaction domain; violet box, TPR; light green box, DJ-1 domain. C. Domain organization of PUB genes in Arabidopsis, rice and barley 
classes similar to those in Arabidopsis and rice based on the domain structures present.

Functional analyses of PUB genes have mainly been conducted in Arabidopsis and rice, and document that the encoded PUB proteins play important roles in plant adaptation and response to many environmental stresses, including drought and microbial attack. For instance, the ubiquitination pathway has been implicated in both ABA-dependent and ABA-independent drought responses. Arabidopsis AtPUB18 and AtPUB19 are strongly up-regulated in response to abscisic acid (ABA) and mutation studies showed that the encoded proteins act as negative regulators of ABA-mediated drought responses [17] whereas the proteins AtPUB22 and AtPUB23 were shown to act as negative regulators of ABA-independent drought responses [18]. Arabidopsis AtPUB18 and AtPUB19 were also suggested to function as regulatory components in salt inhibited germination [19] and AtPUB30 has been suggested to function as a negative regulator of salinity tolerance because loss of function mutants exhibited increased salt stress tolerance in the germination stage [20]. Rice, OsPUB2 and OsPUB3 apparently interact to form heterodimeric complexes and are involved in positive regulation of low temperature stress [21]. Phosphate starvation leads to strong up-regulation of rice OsUPS (OsPUB41), suggesting an important role of OsPUB41 in the Pi signaling pathway [22]. Several PUBs also play distinctive roles affecting plant growth and development. Arabidopsis SAUL1 (AtPUB44) controls leaf senescence and enhances cell death in different tissues [23], whereas AtPUB4 functions as a global regulator of asymmetric cell divisions and cell proliferation during root development [24] and rice TUD1 (OsPUB75) regulates brassinosteroid-mediated growth [25].

Some PUBs have been implicated in both abiotic and biotic stress responses. Together AtPUB24, AtPUB22 and AtPUB23 are involved in PAMP-triggered immunity (PTI) towards microbials [26]. Likewise, rice SPL11 (OsPUB11) and its Arabidopsis orthologs AtPUB12 and AtPUB13 were shown to negatively regulate innate immunity and defense responses [27-29] by their ability to ubiquitinate the receptor-like kinase FLS2 (Flagellin Sensing 2) protein after bacterial infection. OsPUB11 ubiquitinates SPIN6 (a Rho GTPase-activating protein) controlling disease resistance signaling during both fungal and bacterial infection [27-29]. OsPUB44 positively regulates peptidoglycan- and chitin-triggered immunity and resistance to the bacterium Xanthomonas oryzae [30] and OsPUB15 interacts with the receptor-like kinase PID2 to regulate cell death and immunity against rice blast [31]. AtPUB17 is a functional homolog of tobacco ACRE276, improving race-specific resistance against Avr9 from the pathogenic fungus Cladosporium fulvum and against the Gram-negative bacterium Pseudomonas syringae [32]. The potato homolog StPUB17 was shown to promote specific immune pathways triggered by Phytophthora infestans [33] suggesting a conserved function as positive regulators of cell death and defense for these Class II ARM repeat E3 ligases. Beside the rice PUB genes, only two other cereal PUB genes have been functionally characterized. Wheat TaPUB1 was shown to modulate drought stress responses by modulating the antioxidant capability [34] and CMPG1-V from the diploid wheat relative Haynaldia villosa $\mathrm{L}$. was shown to increase resistance against the powdery mildew fungus [35].

In this study, we have identified the members of the PUB protein family in barley based on the published high-quality reference genome sequence of barley (Hordeum vulgare) [36]. Using the available annotation, Hidden Markov Model genomic analysis and blast searches with Arabidopsis and rice PUB protein sequences we identified $67 H v P U B$ genes. Sequence alignments, domain patterns and phylogenetic analyses of the barley, Arabidopsis and rice PUB proteins revealed that the previous classification of PUB genes in Arabidopsis and rice has an ambiguity in the grouping of the Class III ligases [8]. We propose a re-classification of the Arabidopsis, rice and barley PUB proteins into 10 Classes according to their functional domains using NCBI CDD (conserved Domain Database) and InterPro protein domain predictions. The potential involvement of the predicted HvPUB genes in abiotic and biotic stress responses was investigated by analyzing public available full length cDNA and EST libraries and by expression profiling of the $H v P U B$ genes under drought stress or during attempted infection by the powdery mildew fungus.

\section{Results}

\section{Identification of U-box E3 ligase encoding genes in the barley genome}

Based on the published high-quality reference genome sequence of barley (Hordeum vulgare) [36], BLAST searches using full length cDNA sequences and ESTs encoding U-box-type E3 ligases in Arabidopsis and rice as query sequences, $67 \mathrm{U}$-box E3 ligase encoding genes were predicted in barley (Fig. 1a, c). In agreement with current terminology the genes were termed $H v P U B$ genes (Table 1). Mapping the genome loci onto the chromosomes shows that the HvPUB genes are distributed between all the chromosomes (Table 1). In a few places, two or more HvPUB genes are arranged tandemly or closely clustered together. HvPUB11/12 locate in tandem and have $100 \%$ identical sequences, and this is also true for $H v P U B 58 / 59$, indicating recent gene-duplications. However, the gene pairs such as $H v P U B 6 / 43 /$ 52, HvPUB13/25, HvPUB15/16 and HvPUB28/29 are different. Even though the genes map cluster together, they 
Table 1 List of 67 named barley PUB genes with their classification, genome locus, and the number of matching ESTs from libraries categorized as originated from either abiotic- or biotic stress conditions or from vegetative or generative tissue

\begin{tabular}{|c|c|c|c|c|c|c|c|}
\hline \multirow[t]{3}{*}{ Class } & \multirow[t]{3}{*}{ Name } & \multirow[t]{3}{*}{ Gene ID } & \multirow[t]{3}{*}{ Chromosome position } & \multicolumn{4}{|c|}{ No. EST from library conditions } \\
\hline & & & & Abiotic & Biotic & Generative & Vegetative \\
\hline & & & & stresses & & & \\
\hline । & HvPUB1 & HORVU7Hr1G108540 & chr7H:625630612-625,636,687 & 3 & & 18 & 14 \\
\hline$\| \mathrm{a}$ & HvPUB2 & HORVUOHr1G020210 & chrUn:105982242-105,985,875 & 14 & & 4 & 3 \\
\hline Ila & HvPUB3 & HORVU1Hr1G069990 & chr1H:487882055-487,884,794 & 25 & & 16 & 7 \\
\hline Ila & HvPUB4 & HORVU2Hr1G068080 & chr2H:478112149-478,116,380 & & & 2 & \\
\hline$\| \mathrm{a}$ & HvPUB5 & HORVU2Hr1G084670 & chr2H:612532269-612,544,059 & & & & \\
\hline$\| \mathrm{a}$ & HvPUB6 & HORVU2Hr1G107270 & chr2H:711398163-711,399,326 & 2 & & 8 & 3 \\
\hline Ila & HvPUB7 & HORVU3Hr1G081300 & chr3H:594390155-594,392,901 & 8 & & & 2 \\
\hline$\| \mathrm{a}$ & HvPUB9 & HORVU3Hr1G113910 & chr3H:689573848-689,580,280 & 8 & 1 & 2 & 11 \\
\hline Ila & HvPUB10 & HORVU4Hr1G059610 & chr4H:497914351-497,923,545 & 7 & 1 & 8 & 10 \\
\hline Ila & HvPUB11 & HORVU5Hr1G021270 & chr5H:102370756-102,375,777 & 20 & 2 & 22 & 12 \\
\hline Ila & HvPUB12 & HORVU5Hr1G021280 & chr5H:102438361-102,442,824 & 19 & 2 & 15 & 10 \\
\hline$\| \mathrm{a}$ & HvPUB13 & HORVU5Hr1G059280 & chr5H:463207931-463,210,666 & 69 & & 2 & 4 \\
\hline Ila & HvPUB14 & HORVU6Hr1G069010 & chr6H:478128962-478,134,602 & 6 & & 14 & 6 \\
\hline$\| \mathrm{a}$ & HvPUB15 & HORVU6Hr1G072420 & chr6H:503270970-503,276,564 & 9 & & 6 & \\
\hline Ila & HvPUB16 & HORVU6Hr1G073280 & chr6H:507756329-507,760,902 & 18 & & 13 & 9 \\
\hline Ila & HvPUB17 & HORVU7Hr1G000780 & chr7H:1309283-1,315,775 & 8 & 1 & 6 & 14 \\
\hline Ila & HvPUB18 & HORVU7Hr1G047920 & chr7H:162626044-162,632,315 & & & & \\
\hline$\| \mathrm{a}$ & HvPUB19 & HORVU7Hr1G061800 & chr7H:290933777-290,936,462 & 10 & & 3 & \\
\hline Ila & HvPUB20 & HORVU7Hr1G121810 & chr7H:654539257-654,541,940 & & & 3 & 2 \\
\hline$\| \mathrm{a}$ & HvPUB21 & HORVU6Hr1G041430 & chr6H:228200647-228,211,681 & 10 & & 8 & 25 \\
\hline Ilb & HvPUB22 & HORVU2Hr1G067610 & chr2H:473427629-473,435,416 & & & & \\
\hline $1 \mathrm{lb}$ & HvPUB24 & HORVU3Hr1G083500 & chr3H:603823686-603,825,481 & 2 & & 2 & 4 \\
\hline $11 \mathrm{~b}$ & HvPUB25 & HORVU5Hr1G029950 & chr5H:183287072-183,291,627 & 19 & 1 & 13 & 32 \\
\hline $11 \mathrm{~b}$ & HvPUB26 & HORVU5Hr1G059910 & chr5H:467836849-467,839,703 & 2 & & 2 & \\
\hline $11 \mathrm{~b}$ & HvPUB28 & HORVU7Hr1G039760 & chr7H:105941903-105,943,737 & 5 & & 2 & \\
\hline IIb & HvPUB29 & HORVU7Hr1G040790 & chr7H:111351005-111,354,169 & 3 & 1 & 3 & 2 \\
\hline$\| \mathrm{b}$ & HvPUB57 & HORVU6Hr1G066870 & chr6H:463530777-463,532,567 & & & & \\
\hline IIb & HvPUB60 & HORVU6Hr1G095130 & chr6H:583093713-583,097,921 & 9 & & & \\
\hline III & HvPUB31 & HORVU4Hr1G070330 & chr4H:574972338-574,976,289 & & & & 2 \\
\hline IV & HvPUB32 & HORVU1Hr1G053270 & chr1H:393963164-393,967,064 & 1 & & 3 & 1 \\
\hline IV & HvPUB33 & HORVU2Hr1G013130 & chr2H:28662118-28,667,022 & 6 & & & 1 \\
\hline IV & HvPUB34 & HORVU4Hr1G017550 & chr4H:78074992-78,084,602 & 2 & 1 & 2 & 1 \\
\hline IV & HvPUB35 & HORVU4Hr1G088650 & chr4H:640685328-640,696,684 & & 1 & 1 & 5 \\
\hline IV & HvPUB36 & HORVU5Hr1G060580 & chr5H:474627227-474,645,070 & 6 & & & 2 \\
\hline IV & HvPUB37 & HORVU5Hr1G077700 & chr5H:553639844-553,654,837 & & & & 1 \\
\hline IV & HvPUB38 & HORVU6Hr1G003590 & chr6H:8039677-8,048,008 & & & 1 & \\
\hline IV & HvPUB39 & HORVU6Hr1G039290 & chr6H:203742583-203,750,519 & & & & \\
\hline IV & HvPUB40 & HORVU6Hr1G064130 & chr6H:433376429-433,381,410 & & 1 & & 2 \\
\hline IV & HvPUB41 & HORVU7Hr1G018750 & chr7H:25113620-25,118,764 & 2 & & 1 & 7 \\
\hline IV & HvPUB42 & HORVU7Hr1G086580 & chr7H:522444326-522,451,758 & 14 & & 11 & 22 \\
\hline V & HvPUB8 & HORVU3Hr1G089040 & chr3H:627083327-627,148,340 & 17 & & 7 & 14 \\
\hline
\end{tabular}


Table 1 List of 67 named barley PUB genes with their classification, genome locus, and the number of matching ESTs from libraries categorized as originated from either abiotic- or biotic stress conditions or from vegetative or generative tissue (Continued)

\begin{tabular}{|c|c|c|c|c|c|c|c|}
\hline \multirow[t]{2}{*}{ Class } & \multirow[t]{2}{*}{ Name } & \multirow[t]{2}{*}{ Gene ID } & \multirow[t]{2}{*}{ Chromosome position } & \multicolumn{4}{|c|}{ No. EST from library conditions } \\
\hline & & & & $\begin{array}{l}\text { Abiotic } \\
\text { stresses }\end{array}$ & Biotic & Generative & Vegetative \\
\hline V & HvPUB23 & HORVU4Hr1G064070 & chr4H:536981409-536,983,117 & 11 & 1 & & \\
\hline V & HvPUB27 & HORVU6Hr1G077120 & chr6H:528582237-528,584,114 & & 4 & 2 & 6 \\
\hline V & HVPUB30 & HORVU7Hr1G046920 & chr7H:155292351-155,294,342 & 6 & & 2 & 6 \\
\hline V & HvPUB43 & HORVU2Hr1G104640 & chr2H:704314927-704,319,431 & 1 & & 1 & \\
\hline V & HvPUB44 & HORVU3Hr1G095960 & chr3H:651507615-651,508,824 & & & & \\
\hline V & HvPUB45 & HORVU5Hr1G005830 & chr5H:9430272-9,431,296 & & & & \\
\hline V & HvPUB46 & HORVU5Hr1G081160 & chr5H:563433753-563,438,456 & & & & \\
\hline V & HvPUB47 & HORVU7Hr1G093780 & chr7H:572254844-572,256,019 & & & & 2 \\
\hline V & HvPUB48 & HORVUOHr1G003810 & chrUn:17443362-17,445,805 & 13 & & & 8 \\
\hline V & HvPUB49 & HORVU1Hr1G074180 & chr1H:507187193-507,189,384 & 2 & & & 6 \\
\hline V & HvPUB50 & HORVU2Hr1G074130 & chr2H:535102978-535,105,576 & 10 & & 10 & 12 \\
\hline V & HvPUB51 & HORVU2Hr1G076470 & chr2H:550697966-550,699,310 & & & & \\
\hline V & HvPUB52 & HORVU2Hr1G105720 & chr2H:707375224-707,377,465 & 2 & & & 5 \\
\hline V & HvPUB53 & HORVU2Hr1G123130 & chr2H:754722899-754,723,432 & 1 & & & \\
\hline V & HvPUB54 & HORVU4Hr1G066070 & chr4H:550510764-550,513,664 & 2 & & 21 & 13 \\
\hline V & HvPUB55 & HORVU6Hr1G034250 & chr6H:159856080-159,857,826 & & & 2 & \\
\hline V & HvPUB56 & HORVU6Hr1G065480 & chr6H:450926585-450,933,083 & 4 & & 2 & \\
\hline V & HvPUB58 & HORVU6Hr1G089560 & chr6H:570052386-570,053,961 & 19 & & & \\
\hline V & HvPUB59 & HORVU6Hr1G089590 & chr6H:570107770-570,109,318 & 14 & & & \\
\hline V & HvPUB61 & HORVU7Hr1G073100 & chr7H:412252306-412,361,703 & 2 & & & 4 \\
\hline $\mathrm{Vl}$ & HvPUB62 & HORVU1Hr1G039050 & chr1H:272999682-273,010,309 & 19 & & 20 & 5 \\
\hline $\mathrm{VI}$ & HvPUB63 & HORVU3Hr1G052520 & chr3H:381485236-381,496,734 & 7 & & 2 & \\
\hline $\mathrm{VI}$ & HvPUB64 & HORVU4Hr1G083960 & chr4H:627004063-627,010,259 & & & 10 & 1 \\
\hline VII & HvPUB65 & HORVU1Hr1G002240 & chr1H:4416074-4,418,966 & 4 & 1 & 1 & 10 \\
\hline VII & HvPUB66 & HORVU7Hr1G076230 & chr7H:445084899-445,090,758 & 7 & & 11 & 3 \\
\hline$x$ & HvPUB67 & HORVU4Hr1G022990 & chr4H:122902796-122,907,027 & 9 & & 8 & 8 \\
\hline
\end{tabular}

are not directly related, indicating that this clustering could be the result of evolutionary selective forces.

\section{Class I and class II U-box E3 ligases}

In Arabidopsis, rice and barley, the Class I E3 ligases are represented by a single protein member. The protein encoded by the barley gene $H v P U B 1$ contains a UFD2 domain and a U-box domain at the $\mathrm{C}$-terminus like the AtPUB1 and OsPUB1 encoded proteins (Fig. 1b, c). For Class II, we found genes encoding 27 barley U-box proteins possessing repeated ARM/HEAT domains (Fig. 1c). The ARM domains of the $27 H \nu$ PUB proteins were highly conserved with only minor variations in the consensus sequences with $11 \sim 48 \%$ identity and 32 68\% similarity (Fig. 2c). When the full amino acid sequences and domain arrangement of the 27 proteins were compared, we recognized that the Class II proteins can be further assorted into two distinctive groups depending on the proximity of the U-box domain to the $\mathrm{N}$-terminal: the one-fourth of the full length. (Fig. 2a). Class II-a contains 19 members with the U-box positioned near the center of each protein and a U-box $\mathrm{N}$-terminal domain (UND) constituting the $\mathrm{N}$-terminal part. All ARM repeats are positioned in the C-terminal half of the proteins. Class II-b contains 7 members with the U-box domain positioned close to the $\mathrm{N}$-terminal and ARM repeats distributed over the remaining part of the protein sequence (Fig. 2a). Phylogenetic analysis of the barley proteins shows that all the Class II-b proteins share common ancestors with Class II-a genes in the same clades (Fig. 2b). Considering that all the proteins in Class II-b are absent in UND region, Class II-b proteins might have diverged from a Class II-a gene by merely losing the UND region. The opposite scenario is 


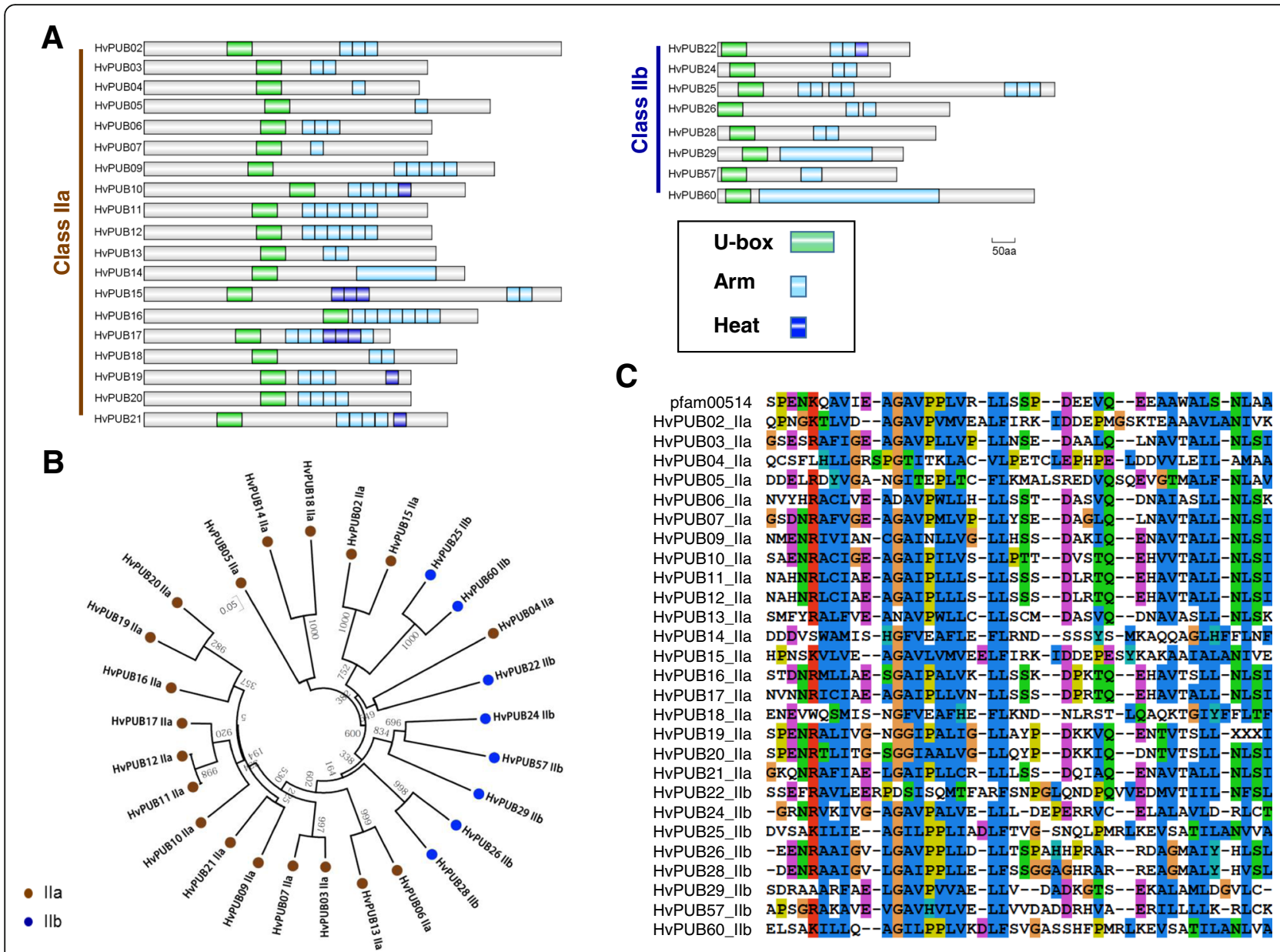

Fig. 2 Domain structures and phylogenetic analysis of Class II genes in barley. a. Domain structures of 23 Class II PUB genes. Green box, U-box domain; sky blue box, ARM repeat domain; blue box, Heat domain. b. Phylogenetic analysis of 23 Class II PUB genes in barley. Brown dot, subclass a; blue dot, subclass b. c. Full-length amino-acid sequences of ARM repeat domain were aligned using the Clustal X2 software. The tree was constructed by neighbor-joining method after bootstrap analysis for 1000 replicates [11]

possible that a common ancestor of Class II protein might do not have UND region and Class II-a acquired the UND region later. However, the consensus sequences of the Class II-b ARM repeats are identical to those of Class II-a, implying that the II-a/II-b evolutionary branching has occurred after integration of the ARM repeats (Fig. 1c) and the branching happened in two different points (Fig. 2b). Therefore, it could be more rational to accept the first scenario to explain the branching of these subgroups. In our study, Arabidopsis has 27 AtPUB genes encoding Class II proteins possessed ARM/HEAT repeats. Based on the presence or absence of the UND domain, 17 belonged to Class II-a and 10 belonged to Class II-b (Additional file 1: Figure $\mathrm{S} 1$ ). In rice 30 of the OsPUB genes possessed ARM/ HEAT repeats and were classified as Class II proteins and 19 belonged to Class II-a and 11 belonged to Class II-b (Additional file 1: Figure S2). The validity of Class II sub-grouping was confirmed by the phylogenetic analysis of all Class II PUB sequences from Arabidopsis, rice, and barley which revealed distinct Class II-a and Class II-b sub-groups (Fig. 3). In general, the Class II PUB sequences are highly conserved between all three species suggesting that all Class II-a and Class II-b sub-groups share common ancestors for all the tested species. This would imply that the Class II subgroups arose before the evolutionary specification of these species (Fig. 3).

\section{Class III, class IV, and class V U-box E3 ligases}

Previously, Zeng et al. suggested that Arabidopsis and rice Class III U-box proteins harbor a putative GKL-box domain in addition to the U-box domain [10] (Fig. 4a). However, we could not find any evidence that supports the functionality of the proposed GKL-box domain in eukaryotes. OsPUB40 has been reported as a protein harboring a GKL-box domain protein [10] but it contains a clear ARM repeat domain in our analysis. Considering that its clustered neighbours OsPUB41, 


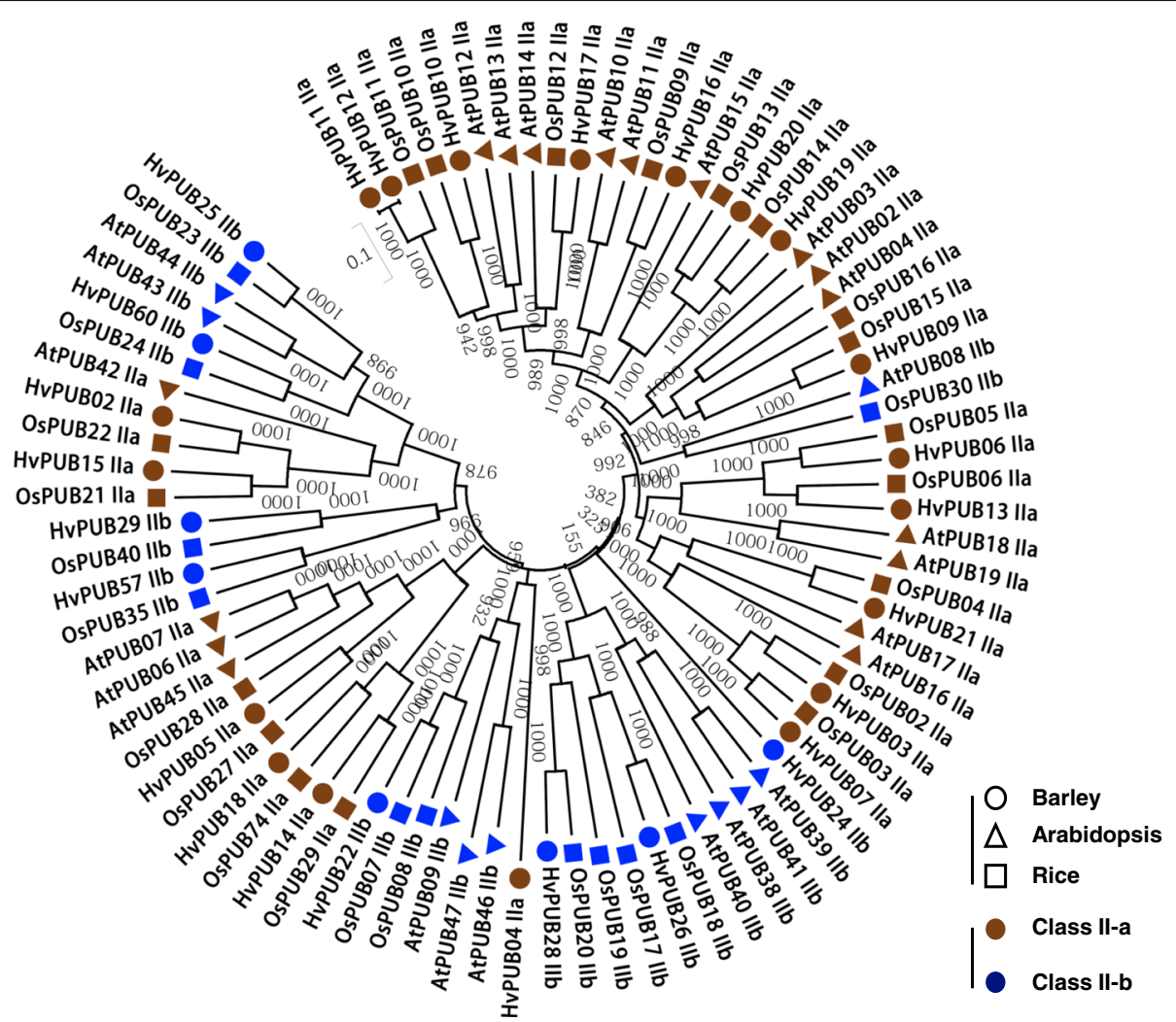

Fig. 3 Phylogenetic tree of Class II genes in Arabidopsis, rice and barely. Brown color indicates Class II-a. Blue color indicates Class II-b. Triangle, Arabidopsis; Circle, barley; Square, rice

OsPUB42, and OsPUB43 do not contain an ARM repeat domain, its presence in OsPUB40 was intriguing (Fig. 4a). This observation led us to search for the ARM repeat sequence of OsPUB40 in other Class III PUBs. Surprisingly, most of the rice Class III PUBs possess a domain which share high sequence homology to an ARM repeat domain albeit with slight aberrancies in the consensus sequences. Therefore, we redefined the GKL-domain as an ARM-like region. Likewise, most of the Class III PUB proteins in Arabidopsis also contained ARM-like regions (Additional file 1: Figure S3). However, ARM-like region cannot be considered as ARM repeats domain. Therefore, we suggest that Class III proteins in Arabidopsis and rice should be regrouped with the Class $\mathrm{V}$ proteins, which characterized by having no distinctive functional domains. In this context, 16 and 20 proteins of Arabidopsis and rice, respectively, were re-classified from Class III to Class V. Based on these criteria, $21 H v$ PUBs genes were assigned to Class V (Fig. 1c, Fig. 4b). Our analyses of the PUB proteins in barley further revealed that the barley HvPUB31 gene encoded a protein with a cyclophilin domain in addition to the U-box domain. This E3 ligase makes a distinct phylogenetic group joined by two orthologues proteins AtPUB49 and OsPUB26 (Fig. 1b and c, Table 1, Fig. 5). Although distinctive, PUB proteins with a cyclophilin domain have not previously been considered as an independent class. Because the peptidyl prolyl isomerase activity of cyclophilin is well-defined in many proteins, we suggest that this type of PUB proteins are combined into a new Class III. Eleven $H \nu \mathrm{PUB}$ proteins were found to contain a kinase domain in addition to the U-box. Class IV PUB proteins contain a PKc (Catalytic domain of the Serine/Threonine kinases) domain at the C-terminal (Additional file 1: Figure S4) and/or an STK_N (N-terminal domain of Eukaryotic Serine Threonine kinases) domain at the $\mathrm{N}$-terminal. Following the previous classification in Arabidopsis and rice [10, 12], we assorted those genes into Class IV. Rice and Arabidopsis and hold 17 and 14 homologue Class IV PUB genes, respectively (Fig. $1 \mathrm{~b}$ and c, Table 1, Fig. 5). We found that Class IV can be grouped into three subgroups; subgroup I has both kinase domains, while subgroup II has six genes with only PKc domain and subgroup III has five genes with only STK-n domain (Additional file 1: Figure S4). Phylogenic analysis, according to their whole sequence homology, not by the kinase domain compositions, showed that the subgroup III might be branched by simply losing the PKc domain, which only happened in Arabidopsis. Besides, 

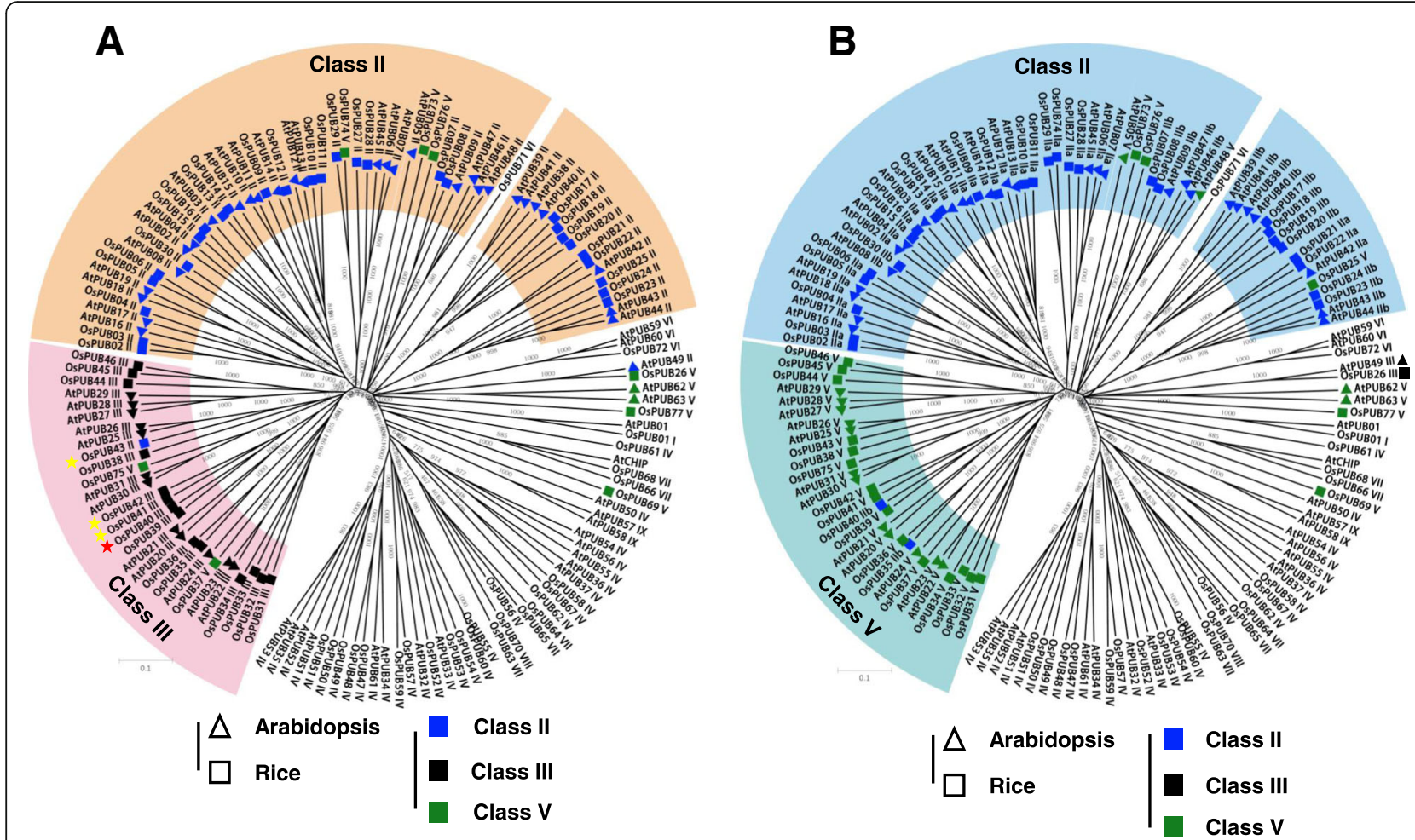

Fig. 4 Phylogenetic analyses of PUB genes in Arabidopsis and rice. a. Phylogenetic analysis based on the classification category of previous studies [10]. In this phylogenic analysis, GKL-domain proteins were classified as Class III. Yellow asterisks indicated genes without ARM repeat domain in rice, OsPUB41, OsPUB42, OsPUB43. Red asterisk indicates OsPUB40 with ARM repeat domain. $\mathbf{b}$. Phylogenetic analysis based on the renaming category that was suggested in this study. Most of Class III genes in Arabidopsis and rice were renamed into Class V except OsPUB35 and OsPUB40 that were sorted into Class II

subgroup II seemed to be randomly branched from subgroup I of rice and barley (Additional file 1: Figure S5).

\section{The other classes of U-box E3 ligases}

Previously, PUB genes that encode proteins with a $\mathrm{U}$-box at the N-terminal and a WD40 domain at the C-terminal were classified as Class VI [10]. We found that barley has three $H v P U B$ genes encoding proteins belonging to Class VI (Fig. 1b and c, Table 1, Fig. 5). The domain organization in $H \nu$ PUB63 is different from $H \nu$ PUB62 and $H v$ PUB64 even though the three genes are assigned into Class VI (Additional file 1: Figure S6). We further identified two TRP domain containing PUB genes and assorted them as Class VII in barley (Fig. 1b and c, Table 1, Fig. 5). Arabidopsis and rice have a single and five members of Class VII. In Arabidopsis, two AtPUB proteins have a MIF4G domain at the $\mathrm{N}$-terminal region in addition to a U-box domain. These Arabidopsis specific genes were classified into Class IX (Fig. 1b and c, Table 1). Class VIII is specific for rice, containing one-member protein which contains a TRP domain and a kinase domain. In addition, our analysis revealed a barley specific U-box protein, HvPUB67, which at the $\mathrm{N}$-terminal region harbours a DJ-1 protein domain for thiamin biosynthesis. Because of the domain specificity, we assigned the DJ-1 containing PUB E3 ligase to a new class, Class X (Fig. 1b and c, Table 1, Fig. 5).

\section{Phylogenetic relationship of PUB proteins in Arabidopsis,} rice, and barley

Our phylogenetic analysis of barley, rice and Arabidopsis showed that most PUB proteins have much closer phylogenetic relationships within their clustered orthologues from other species than their paralogues (Fig. 5). This distinctive grouping among the different classes of PUB proteins across species implied that PUB proteins have largely diversified from a common ancestor before the evolutionary branching of monocots and dicots. For instance, Class II and V proteins were highly clustered among Arabidopsis, rice and barley (Fig. 5) suggesting that Class II and Class V might share common ancestors which harbor an ARM repeat domain, then they diverged into two distinctive groups, resulting in one group maintaining an ARM repeat domain and the other losing the consensus sequences of the domain. Class IV proteins were also highly well clustered into three different clades (Fig. 5). Clade I consists of 6 Arabidopsis, 5 rice and 5 barley proteins, whereas Clade II has 1 


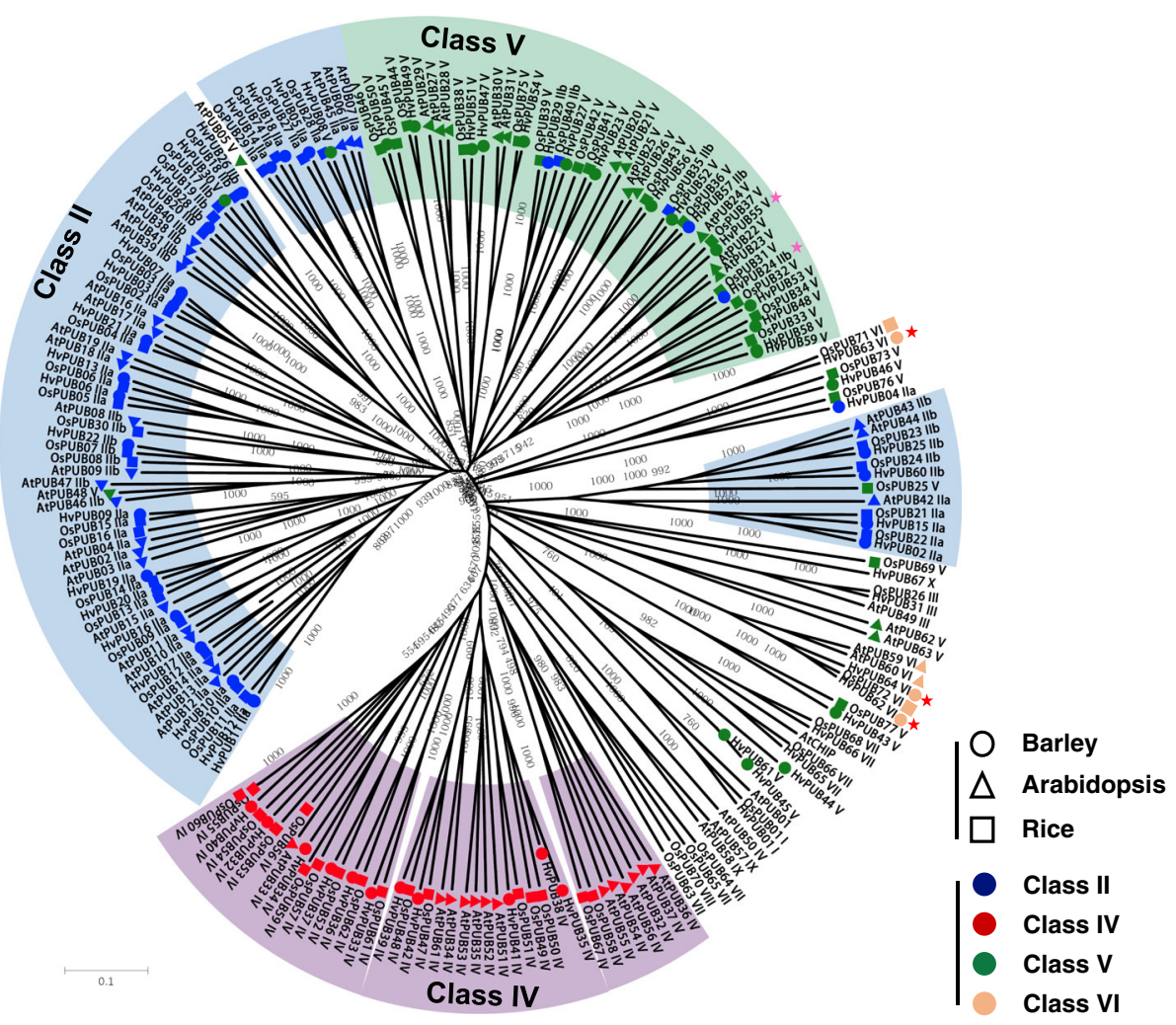

Fig. 5 Phylogenetic analysis of PUB genes in Arabidopsis, rice, and barley. Class II genes were clustered and colored with blue. Class IV genes were clustered and colored with red. Class V genes were clustered and colored with green. Class VI genes were not clustered and indicated with incarnadine colored marks. Red asterisks indicate barley genes in Class VI. Pink asterisks show HVPUB24 and HvPUB55 gene pair

Arabidopsis, 9 rice and 6 barley proteins. Clade III does not include barley proteins (Additional file 1: Figure S5). In the case of Class VI, two Arabidopsis proteins (AtPUB59 and AtPUB60), one rice protein (OsPUB72) and two barely proteins ( $H v$ PUB62 and $H v$ PUB64) were well clustered in the clade (Fig. 5). HvPUB63 is positioned in an independent clade together with OsPUB71, which is not related to Class VI. This result implied that the two different clades of Class VI also branched before the speciation of the three species. Furthermore, we found that several $H \nu$ PUB proteins belonging to Class $\mathrm{V}$ are more related to Class I and Class VII than to the largest clade of Class V. For instance, $H v$ PUB44, $H v$ PUB45, and $H v$ PUB61 fall into Class $\mathrm{V}$ based on the module content but form an independent sister taxon, in between Class I genes and Class VII genes. We speculate that the phylogenic distribution of these barley genes might be caused by losing a TRP or UFD encoding domain during their evolution. Similarly, several genes in Arabidopsis and rice may during evolution have lost the sequence encoding the ARM repeat domains. For instance, two Class $\mathrm{V}$ gene in Arabidopsis encoding AtPUB5 and AtPUB48 seemed to have branched from Class II genes. In rice, OsPUB25 may have diverged from
Class II genes. HvPUB8 and HvPUB30 genes also appear to have diverged from Class II genes (Fig. 5). In contrast, we observed that several rice and barley Class II genes may have been reverted from Class V. For example, OsPUB35, OsPUB40, HvPUB24, HvPUB29, and HvPUB57 were clustered with Class $\mathrm{V}$ genes although these genes contain sequences encoding ARM repeats (Fig. 5). As shown here, the classification of PUB proteins in plants solely based on their defined domains imposes ambiguity in assigning the proteins into specific Classes and Clades. However, most of the PUB proteins in barley show similar phylogenetic distributions to those in Arabidopsis and rice, implying that $P U B$ genes are evolutionarily well conserved across dicots and monocots.

\section{Expression profile of $H v P U B$ genes in response to drought stress}

$P U B$ genes are negatively or positively involved in plant responses to drought stress. The expression profiles of the majority of the identified barley $H v P U B$ genes as response to drought stress were investigated by subjecting two-weeks-old barley seedlings to abrupt dehydration conditions. The onset of drought responses in the seedling plants was monitored as induced expression of the 
well-defined drought induced gene Dehydrin1 (Deh1) (Fig. 6a). In the drought stress treated seedlings, Deh1 expression increased 8-fold compared to the nonstressed control seedlings.

As shown in Fig. 6, the expression of the barley $H \nu P U B$ genes were dynamically altered by the drought stress. A subset of the genes showed two-fold increase in expression when compared to the control plants. Significant changes in expression after drought stress were observed for HvPUB genes from Class II, Class VI and Class VI. Within Class II, expression of five genes, HvPUB7, HvPUB9, HvPUB15, HvPUB16, HvPUB21 and $H v P U B 22$ were notably induced, whereas expression of HvPUB18 was strongly reduced (Fig. 6c). Within Class $\mathrm{IV}$, the expression of four genes, HvPUB32, HvPUB34, $H v P U B 37$, and HvPUB40 was induced by around two-fold (Fig. 6d). The expression of the HvPUB64 gene from Class VI was increased over two-fold (Fig. 6f). In Class V and Class VII, none of the genes was significantly up-regulated by drought stress (Fig. $6 \mathrm{~g}$ and e). The expression of 11 out of 67 barley $H v P U B$ genes was at least two-fold induced in seedlings under the drought treatment and the expression of the gene $H v P U B 18$ was suppressed, supporting the strong involvement of the
PUB gene family in plants drought stress responses (Fig. 6, Additional file 1: Figure S7).

\section{Expression profile of HVPUB genes in response to fungal infection}

PUB genes are also known to play a regulatory role in plant responses to pathogen stress. Changes in the expression profile of $H v P U B$ genes in response to fungal infection was investigated using barley powdery mildew as the experimental system. Two weeks-old barley seedlings were inoculated with Blumeria graminis f.sp. hordei $(B g h)$ spores and $18 \mathrm{~h}$ after inoculation, the expression of the two pathogen responsive marker genes $H v P R 1 b$ and $H v P R X 8$ was monitored to ascertain the activation of pathogen stress responses. Expression of $H v P R 1 b$ and $H v P R X 8$ was induced by around 170 and 60 -fold, respectively, compared to uninfected control plants (Fig. 7a). Overall, the expression of the tested $H v P U B$ genes was suppressed after fungal infection. Only expression of the Class II gene $H v P U B 24$, the Class IV gene $H v P U B 42$ and the Class $\mathrm{V}$ genes $H v P U B 23,27$ and 55 showed a significant 2-fold or higher induction (Fig. 7c, d, and e). The strongest suppression of expression (more than 3-fold) was observed for the Class II

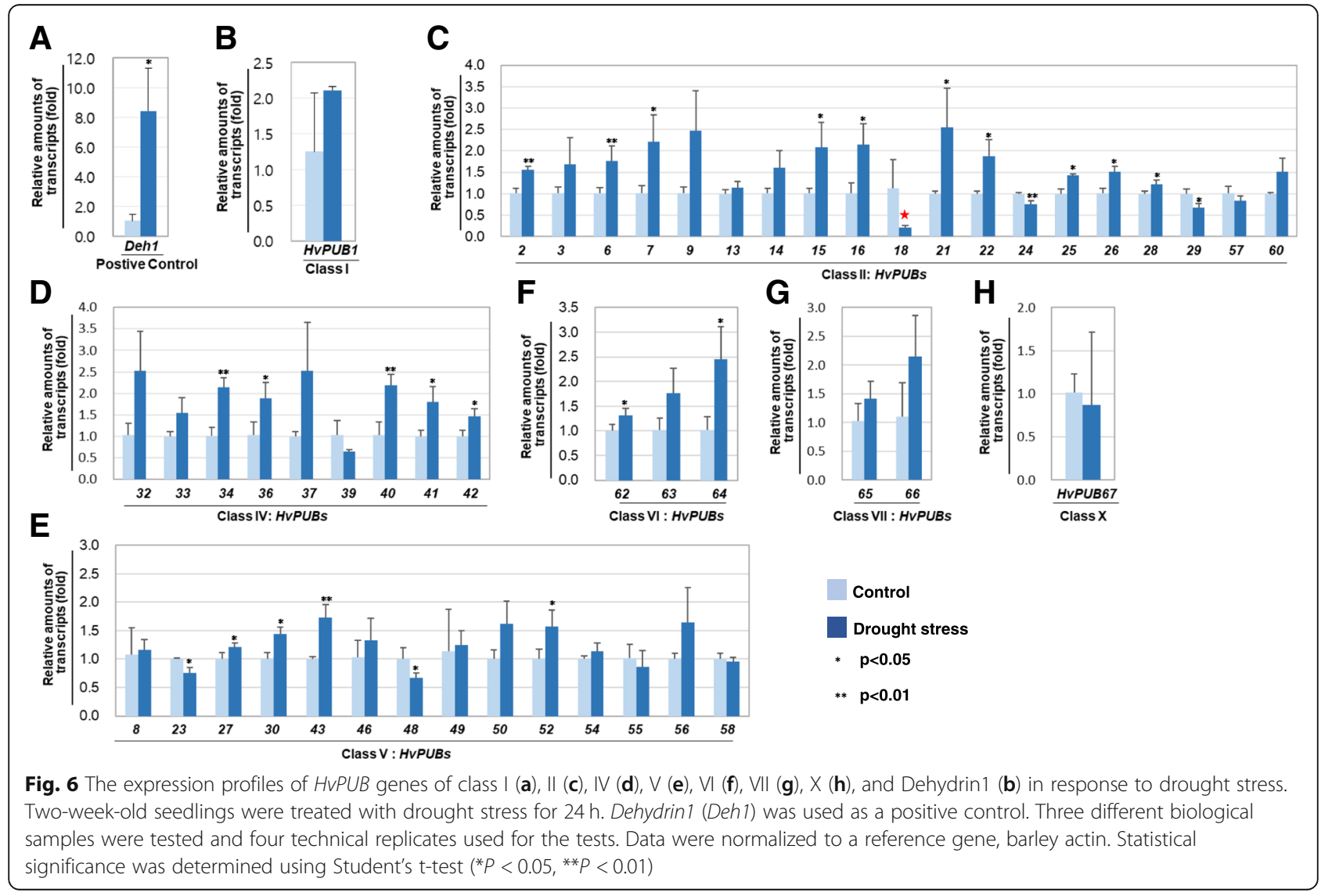




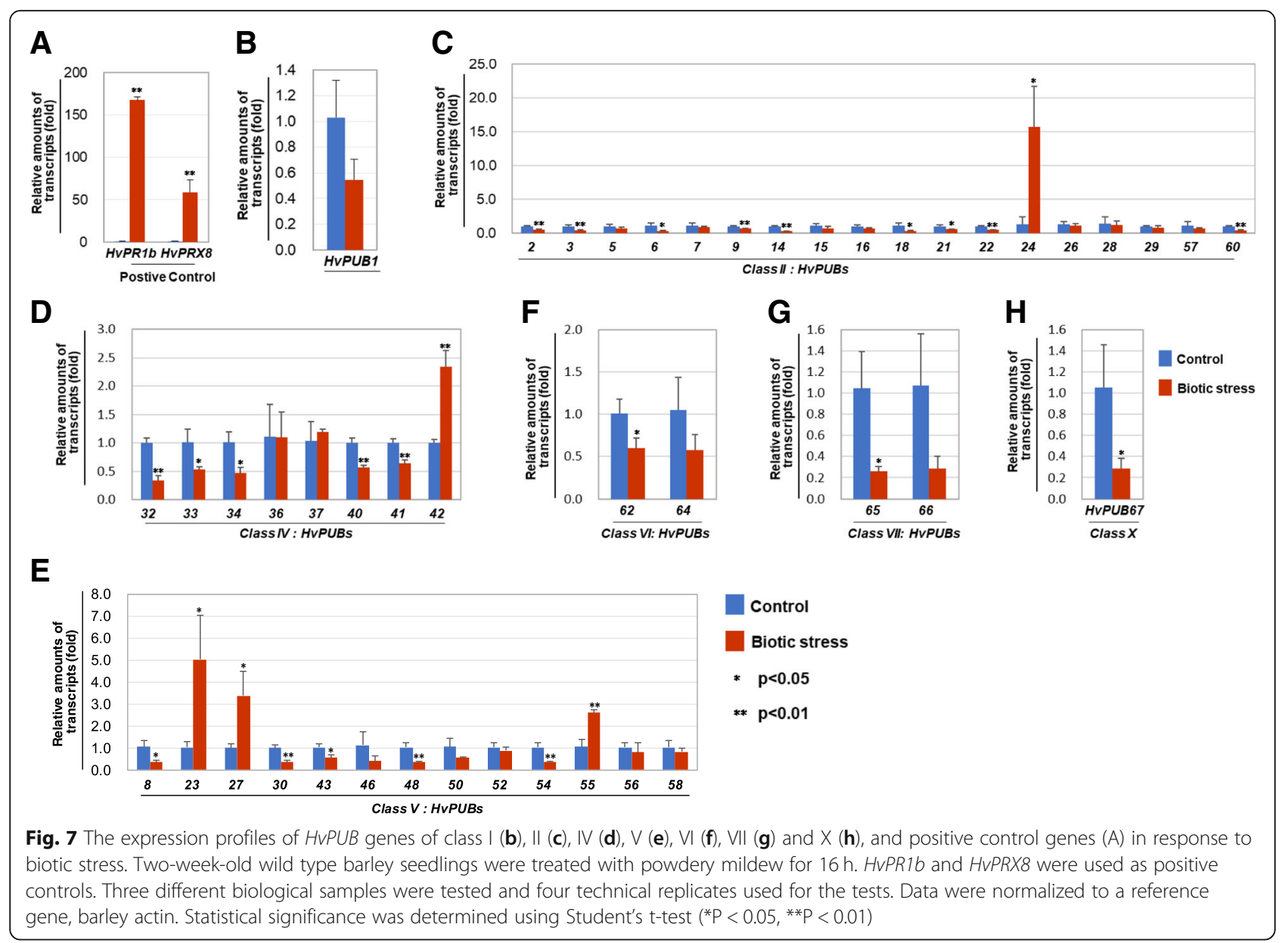

genes HvPUB6, 14 and 18, the Class IV gene HvPUB32, the Class V genes $H v P U B 8,30,46,48$ and 54, the Class VII genes HvPUB65 and 66 and the Class $\mathrm{X}$ gene HvPUB67 (Fig. 7, Additional file 1: Figure S8). In general, the observed changes in expression were not only larger after pathogen stress than the changes observed following drought stress, but they also involved different $H v P U B$ genes. Only HvPUB18 was regulated similarly, i.e. suppressed expression in response to both stress conditions, implying that it might be a negative regulator for both types of stress.

\section{Discussions}

In Arabidopsis and rice, PUB genes are known to play important roles in the plant responses against abiotic and biotic stresses. Functional analysis has revealed detailed molecular mechanisms involving PUB proteins in relation to abiotic stresses and biotic stresses [6]. However, the PUB gene family in barley has so far not been investigated or implicated in barley stress responses. Our analysis identified 67 PUB genes in the barley genome. Based on domain structures and evolutionary relationship, the Arabidopsis, rice and barley $P U B$ genes were categorized into ten different classes. This classification represents a refinement of the previously reported classifications of $P U B$ genes in Arabidopsis and rice [10, 12]. Arabidopsis ARM repeat containing PUB proteins have previously been divided into two sub-groups based on the presence or absence of a UND (U-box N-terminal domain) region [7]. We adapted this in our classification as Class II-a with UND and Class II-b without the UND region. The UND region does not affect the E3 ligase activity, but was shown to determine the ubiquitination specificities of AtPUB18 [37]. No function of the UND region has yet been reported for rice or barley. Based on the phylogenetic analysis it seems that Class II-b PUB genes from the analyzed species have evolved from common ancestral genes rather than from Class II-a PUB genes. Previous studies suggested that $28 P U B$ genes in rice and 29 PUB genes in Arabidopsis belong to Class II [10]. However, bioinformatic analysis using the most recent sequence data, we redistributed several of the previously assigned Class II genes of Arabidopsis and rice into other classes. For instance, we could not confirm the presence of sequences encoding ARM/HEAT repeats in two Arabidopsis (AtPUBO5 and AtPUB48) and three 
rice genes (OsPUB25, OsPUB73, and OsPUB76). Accordingly, these genes were therefore transferred into Class $\mathrm{V}$ among the other PUB proteins without extra subdomains (Fig. 4, Additional file 1: Figure S1 and S2).

Zeng et al. [10] identified a motif with highly conserved glycine, lysine/arginine and leucine rich residues in the C-terminal part of several Arabidopsis and rice PUB proteins and named this region as a GKL-domain. Based on the presence of this putative domain, these PUB proteins were placed in Class III, independent on the presence of other domains. However, our analyses revealed that the GLK domain that formed the basis for the grouping of certain PUB proteins in Class III exhibited a high degree of sequence similarity to the ARM repeats. Re-analysis of the Arabidopsis and rice PUB proteins assigned to Group III by Zeng et al. demonstrated that AtPUB21, AtPUB30, OsPUB39, and OsPUB40 contain obvious ARM repeat domains. In the other PUB proteins classified to Group III, a few of the conserved residues of the ARM domain were missing. We therefore decided not to classify these PUBs with ARM-like region to Class II but instead assigned these sequences to Class $\mathrm{V}$ for which the common denominator is the absence of other domains than the U-box (Additional file 1: Figure S3). In the study of Zeng et al., AtPUB49 and OsPUB26 were classified as Class II and Class V proteins, respectively. We found that both proteins contain a cyclophilin domain in addition to the U-box domain and discovered that the barley protein (HvPUB31) also harbored such a domain. We argue that the presence of a cyclophilin domain with peptidyl prolyl isomerase activity is so significant that it justifies the assignment of these proteins to a re-defined Class III. Our analysis also showed that five Arabidopsis genes (AtPUB36, AtPUB37, AtPUB54, AtPUB55, and AtPUB56) previously classified to Class $\mathrm{V}$ possess a clear serine/threonine kinase domain $[24,26]$. Therefore, we re-classified these genes into Class IV (Fig. 5, Additional file 1: Figure S4). Compared to the classification of PUB proteins in Arabidopsis and rice, two classes (Class VIII and Class IX) are absent in barley. Rice OsPUB70 harbors a TRP as well as a kinase domain and is classified as Class VIII, while two Arabidopsis PUB proteins, AtPUB57 and AtPUB58, with MIF4G domains are classified as members of Class IX. Instead, barley has a unique U-box E3 ligase, $H \nu$ PUB67 which contains a DJ-1 domain with a U-box domain and is the only member of Class X. Interestingly, searching other plant genomes we could not find a ortholog of this protein. This suggests that $H v$ PUB67 is a unique barley protein.

Our investigation showed that most barley $H v P U B$ genes were regulated in response to fungal attack by $B g h$ or in response to drought (Figs. 6 and 7). Five HvPUB genes were up regulated in response to $B g h$ attack, whereas, expression of the majority of the remaining $H v P U B$ genes were down regulated in response to $B g h$. In response to the imposed drought treatment, 11 $H v P U B$ genes were activated and only one was down regulated. Except for the down regulation of HvPUB18 under both biotic and drought stress, we did not observe any overlap in or contrasting regulation of the $H v P U B$ genes, although a dual role of both abiotic and biotic stresses has been reported for many of the functionally characterized Arabidopsis and rice PUB genes $[18,26]$. Arabidopsis AtPUB2O (alternatively known as AtCMPG1) is immediately induced by an elicitor, indicating a role in the early steps of pathogen-host interactions in Arabidopsis [38]. In tobacco and tomato, CMPG1 genes orthologous to AtPUB2O are induced by elicitor and wounding treatments in a typical $A C R E$ gene expression pattern. In tobacco, a deficiency in NtPUB20 gene expression compromises the immunity of the plants [39]. AtPUB20 is able to directly interact with the G-protein b-subunit, AGB1, but it is unclear whether this protein-protein interaction is relevant to plant defense [40]. Functional characterization of the orthologue CMPG-V from Haynaldia villosa L., a diploid wheat relative, showed that this $P U B$ gene was induced in leaves and stem of $H$. villosa upon inoculation with the wheat powdery mildew fungus Blumeria graminis f. sp. Tritici. Over-expression of CMPG1-V in susceptible wheat lead to improved broad-spectrum powdery mildew resistance [35]. Our analysis showed that HvPUB23 and HvPUB27 are closely related to AtPUB2O and $C M P G 1-V$, with $H v$ PUB27 sharing more than $90 \%$ homology to CMPG1-V at the amino acid sequence level. In agreement with this observation, our fungal infection studies demonstrate that HvPUB23 and HvPUB27 were identified among the five genes showing induced expression after Bgh inoculation (Additional file 1: Figure S8), strongly implying a role of these $H v P U B$ genes in barley powdery mildew resistance. Arabidopsis AtPUB22, AtPUB23, and AtPUB24 function as negative regulators of pathogen-associated molecular patterns (PAMPs)-triggered responses. Upon encountering pathogens, plants recognize PAMPs by pattern recognition receptors [26] Ubiquitination and vesicle trafficking have been linked to the regulation of immune signaling. In the signaling, Exo70B2, a subunit of the exocyst complex is required for both immediate and later responses [41]. AtPUB22 mediates the ubiquitination of Exo70B2 and its subsequent degradation by the $26 \mathrm{~S}$ proteasome to attenuate PAMPinduced signaling [41]. Two close relatives in barley, HvPUB24 and HvPUB55 were up-regulated by fungal infection (Fig. 7c and e). Those genes might share a similar function as regulators of PAMP-triggered responses, as both were found to be induced following Bgh inoculation. Furthermore, a recent study suggested that HvPUB15/ 
ARM1 gene pair is a case of gene neo-functionalization after a non-tandem, partial gene-duplication event that gained a role in quantitative resistance against $B g h$ and maybe other pathogenic fungi [42]. Although HvPUB15 was not significantly up-regulated by $B g h$ infection in our study, the non-tandem and partial gene-duplication of ARM domain in HvPUB24/HvPUB55 gene pair could be another case of neo-functionalization for boosting pathogen tolerance (Fig. 5).

AtPUB22 and AtPUB23 play an essential role in drought responses. Mutations in these two genes have led to increased tolerance against drought stress relative to wild-type plants [18]. AtPUB22 and AtPUB23 conjugate ubiquitins to the $19 \mathrm{~S}$ proteasome regulatory particle (RP) subunit RPN6, resulting in its degradation and altered activity of the $26 \mathrm{~S}$ proteasome in response to drought stress [43]. In our study, the expression of barley HvPUB24 was rather down-regulated and HvPUB55 was not notably affected by drought conditions (Fig. 6). AtPUB17 has been implicated as a positive regulator of plant defense and stress signaling responses [32], whereas the closely related rice orthologues OsPUB2/3 were shown to play a role as positive regulators of temperature stress [21]. Barley has four orthologue genes, HvPUB3, HvPUB7, and HvPUB21, with HvPUB3/ 7 sharing more than $80 \%$ homology at the amino acid level to OsPUB2/3. Expression of the HvPUB3, HvPUB7 and $H v P U B 21$ genes were induced in response to our drought treatment, again suggesting a potential role like OsPUB $2 / 3$ in abiotic stress responses. Wheat TaPUB1 is up-regulated in response to drought and constitutive over-expression of TaPUB1 in tobacco enhanced drought tolerance in the transgenic plants, most likely caused by increased antioxidant capacity mediated by TaPUB1 [34]. Wheat TaPUB1 contains a WD40 domain and shares high homology with Arabidopsis, rice and barley Class VI PUB proteins. The Arabidopsis orthologues are AtPUB59/60, also known as $M A C 3 A$ and $M A C 3 B$, which in Arabidopsis are members of the MOS4-Associated Complex (MAC) that function redundantly in the regulation of plant innate immunity [44], but no role for AtPUB59/60 in drought or other abiotic stresses has been reported. However, $H \nu$ PUB64 shares 96\% homology to TaPUB1 and is among most highly induced barley $H v P U B$ genes in response to drought, implying a similar role of HvPUB65 in mediating drought tolerance in barley as TaPUB1 in wheat.

\section{Conclusions}

From the current knowledge of the molecular and biological functions of $P U B$ genes it seems that they possess a high degree of conserved functions in plant responses to biotic and abiotic stresses across species. Our data show that many barley PUB genes are also involved in responses against pathogen and drought stresses as negative or positive regulators. Although we showed that many barley $P U B$ genes are transcriptionally regulated by the stresses, further investigations are needed to understand the molecular functions of barley $P U B$ genes and their target proteins to shed further light over stress-response mechanisms in barley.

\section{Methods}

\section{Plant materials and stress treatments}

Barley (Hordeum vulgare L) cv. Golden Promise was from University of Copenhagen, Department of Plant Environmental Sciences, Denmark. It used for drought and pathogen treatments. Plants were grown in a growth chamber at $20^{\circ} \mathrm{C}$ with a photoperiod of $16 \mathrm{~h}$ $\left(200 \mu \mathrm{mol} \mathrm{m}{ }^{-2} \mathrm{~s}^{-1}\right)$.

\section{Drought treatment}

Plants were grown in vermiculite for 2 weeks. The drought treatment was initiated by careful removal of the vermiculite attached to the roots and wrapping the roots in dry soft tissue paper to avoid any influence of light. Sampling took place when the leaves were observed to lose turgor pressure, approximately $24 \mathrm{~h}$ after imposure to the drought stress. Control plants were kept in watered in vermiculite.

\section{Powdery mildew infection}

Plants were grown in a mixture of peat with granulated clay (Pindstrup substrate no. 6; Pindstrup Mosebrug A/S). Spores from Blumeria graminis $\mathrm{f}$. sp. hordei (Bgh) Race A6 were inoculated onto the adaxial side of the second leaf with a density of 80 conidia $\mathrm{mm}^{-2}$. Samples were taken $18 \mathrm{~h}$ after inoculation (hai).

\section{Sequence analysis}

Barley $H v P U B$ genes were predicted from the barley genome sequence [45], using the available Pfam and InterPro domain predictions [46, 47], blast searches with known AtPUB and OsPUB genes and by using a Hidden Markov Model (HMM) built from an alignment of the AtPUB and OsPUB U-box domains predicted by SMART program (http://smart.embl-heidelberg.de/). Conserved domains of the potential $H v$ PUB proteins were analyzed and confirmed using NCBI CDD (Conserved Domain Database) and InterPro protein domain predictions. Full length cDNA and EST sequences matching the predicted $H \nu$ PUB genes were identified by BLAST searches and their expression patterns assessed using the Barley Gene Expression Database (http://barleyflc.dna.affrc.go.jp/bexdb/) [48] and NCBI. Analysis of alignment and phylogenetic tree of full length or U-box 
domain sequences was performed by MEGA7 [49] or CLC workbench software.

\section{Phylogenetic analysis}

The multiple sequence alignments of barley u-box E3 ligase proteins were performed to construct a phylogenetic trees by Clustal X version 2.1 [11]. All phylogenetic trees were constructed according to the using default settings and 1000 bootstrap replications were used to estimate the accuracy of trees.

\section{Real-time qRT-PCR analyses}

Total RNA was isolated from drought-treated and powdery mildew inoculated two-week-old barley seedlings using Spectrum $^{\text {Tw }}$ Plant Total RNA Kit (Sigma). First-strand cDNA was synthesized from $2 \mu \mathrm{g}$ total RNA using the iScript ${ }^{\text {tm }}$ cDNA Synthesis Kit (BioRad). Real-Time qRT-PCR was performed using the CFX384 Touch $^{\text {Tx }}$ Real-Time PCR Detection System (Bio-Rad) with DyNAmo Flash SYBR Green qPCR Kit (Thermo). Each qRT-PCR reaction was performed in technical triplicate using the barley u-box primers in this analysis (Additional file 2: Table S1.) All qRT-PCR data was analysed by using CFX Manager Version 3.1 software (Bio-Rad). Relative gene expression fold was calculated by delta-delta Ct method and the error bars means the \pm standard deviation over technical triplicates of $\mathrm{Cq}$ values.

\section{Additional files}

Additional file 1: Figure S1. Domain structures and phylogenetic analysis of Class II genes in Arabidopsis. A. Phylogenetic analysis of 27 Class II PUB genes in barley. Brown dot, subclass a; blue dot, subclass b. B. Full-length amino-acid sequences of ARM repeat domain were aligned using the Clustal X2 software. The tree was constructed by neighborjoining method after bootstrap analysis for 1000 replicates [1]. C. Domain structures of 27 Class II PUB genes. Green box, U-box domain; skyblue box, ARM repeat domain; blue box, Heat domain. Figure S2. Domain structures and phylogenetic analysis of Class $\|$ genes in rice. A. Phylogenetic analysis of 25 Class II PUB genes in barley. Brown dot, subclass a; blue dot, subclass b. B. Full-length amino-acid sequences of ARM repeat domain were aligned using the Clustal X2 software. The tree was constructed by neighbor-joining method after bootstrap analysis for 1000 replicates [1]. C. Domain structures of 25 Class II PUB genes. Green box, U-box domain; skyblue box, ARM repeat domain; blue box, Heat domain. Figure S3. Class III genes in Arabidopsis and rice, those were converted to new classes, Class II and Class V. Figure S4. Schematic domain structures of Class IV genes in Arabidopsis, rice and barley. Figure S5. Phylogenetic analysis of Class IV PUB genes in Arabidopsis, rice and barely. Figure S6. Domain structures of ClassVI PUB genes in Barley. Figure S7. The expression profiles of HVPUB genes in response to drought stress. A. HvPUB genes are up-regulated by drought stress. B. HVPUB genes are down-regulated by drought stress. Figure $\mathbf{5 8}$. The expression profiles of HVPUB genes in response to biotic stress. A. HvPUB genes are up-regulated by biotic stress. B. HVPUB genes are downregulated by biotic stress. (DOCX $3262 \mathrm{~kb}$ )

Additional file 2: Table S1. List of primers and sequence information. (DOCX $17 \mathrm{~kb})$

\section{Abbreviations}

ABA: abscisic acid; ARM: Armadillo; CHIP: Carboxyl terminus of HSC70interacting protein; Hv: Hordeum vulgare; PAMPs: pathogen-associated molecular patterns; PUB: PLANT U-box; UND: U-box N-terminal domain; UPS: ubiquitin proteasome system

\section{Acknowledgments}

Not applicable.

\section{Funding}

This work was supported by grants from the VILLUM Research Center "Plant Plasticity"; the UCPH Excellence Programme for Interdisciplinary Research to Center for Synthetic Biology, the Korea National Center for Next-Generation BioGreen21 Program (PJ01194601), the National Research Foundation (NRF2016K2A9A1A06922119, NRF-2018R1A6A1A03025607, NRF-

2017R1A6A3A11035981, and NRF-2017R1A2B4010255), Innovation Fund Denmark (6148-00006B)

\section{Availability of data and materials}

Gene sequence information of U-box E3 ligases in barley is available at the Barley Gene Expression Database (http://barleyflc.dna.affrc.go.jp/bexdb/).

\section{Authors' contributions}

SKC, MYR, WTK, LMF and YSW managed this project. SKC and MYR performed experiments. YC, and EK prepared barley samples. SKC, MYR, YH, JK, JHK, GMK, BLM, MFL, and SWY analyzed the data. BLM, MFL, and SWY wrote the manuscript. All authors have read and approved the manuscript.

\section{Ethics approval and consent to participate}

Not applicable.

\section{Consent for publication}

Not applicable.

\section{Competing interests}

The authors declare that they have no competing interests.

\section{Publisher's Note}

Springer Nature remains neutral with regard to jurisdictional claims in published maps and institutional affiliations.

\section{Author details}

'Plant Biochemistry Laboratory, Department of Plant and Environmental Sciences, University of Copenhagen, Thorvaldsensvej 40, DK-1871 Frederiksberg C, Copenhagen, Denmark. ${ }^{2}$ Department of Systems Biology, College of Life Science and Biotechnology, Yonsei University, Seoul 120-749, Korea. Institute of Life Science and Biotechnology, Yonsei University, Seoul, South Korea. ${ }^{4}$ Samsung Medical Center, 81 Irwon-Ro Gangnam-gu, Seoul, Korea.

Received: 20 July 2018 Accepted: 15 April 2019

Published online: 29 April 2019

\section{References}

1. Vierstra RD. The ubiquitin-26S proteasome system at the nexus of plant biology. Nat Rev Mol Cell Biol. 2009;10(6):385-97.

2. Smalle J, Vierstra RD. The ubiquitin 265 proteasome proteolytic pathway. Annu Rev Plant Biol. 2004;55:555-90.

3. Hua Z, Vierstra RD. The cullin-RING ubiquitin-protein ligases. Annu Rev Plant Biol. 2011;62:299-334.

4. Glickman MH, Adir N. The proteasome and the delicate balance between destruction and rescue. PLoS Biol. 2004;2(1):E13.

5. Callis J. The ubiquitination machinery of the ubiquitin system. Arabidopsis Book. 2014;12:e0174.

6. Yee D, Goring DR. The diversity of plant U-box E3 ubiquitin ligases: from upstream activators to downstream target substrates. J Exp Bot. 2009;60(4): 1109-21.

7. Andersen P, Kragelund BB, Olsen AN, Larsen FH, Chua NH, Poulsen FM, Skriver K. Structure and biochemical function of a prototypical Arabidopsis U-box domain. J Biol Chem. 2004;279(38):40053-61. 
8. Wiborg J, O'Shea C, Skriver K Biochemical function of typical and variant Arabidopsis thaliana U-box E3 ubiquitin-protein ligases. Biochem J. 2008;413(3):447-57.

9. Yan J, Wang J, Li Q, Hwang JR, Patterson C, Zhang H. AtCHIP, a U-boxcontaining E3 ubiquitin ligase, plays a critical role in temperature stress tolerance in Arabidopsis. Plant Physiol. 2003;132(2):861-9.

10. Zeng LR, Park CH, Venu RC, Gough J, Wang GL. Classification, expression pattern, and E3 ligase activity assay of rice U-box-containing proteins. Mol Plant. 2008; 1(5):800-15.

11. Larkin MA, Blackshields G, Brown NP, Chenna R, McGettigan PA, McWilliam $H$, Valentin F, Wallace IM, Wilm A, Lopez R, et al. Clustal W and Clustal X version 2.0. Bioinformatics. 2007;23(21):2947-8.

12. Azevedo C, Santos-Rosa MJ, Shirasu K. The U-box protein family in plants. Trends Plant Sci. 2001;6(8):354-8.

13. Riggleman B, Wieschaus E, Schedl P. Molecular analysis of the armadillo locus: uniformly distributed transcripts and a protein with novel internal repeats are associated with a Drosophila segment polarity gene. Genes Dev. 1989;3(1):96-113.

14. Mudgil Y, Shiu SH, Stone SL, Salt JN, Goring DR. A large complement of the predicted Arabidopsis ARM repeat proteins are members of the U-box E3 ubiquitin ligase family. Plant Physiol. 2004:134(1):59-66.

15. Jiao L, Zhang Y, Lu J. Overexpression of a stress-responsive U-box protein gene VaPUB affects the accumulation of resistance related proteins in Vitis vinifera Thompson seedless. Plant Physiol Biochem. 2017;112(Supplement C:53-63.

16. Song J, Mo X, Yang H, Yue L, Song J, Mo B. The U-box family genes in Medicago truncatula: key elements in response to salt, cold, and drought stresses. PLoS One. 2017;12(8):e0182402.

17. Seo DH, Ryu MY, Jammes F, Hwang JH, Turek M, Kang BG, Kwak JM, Kim WT. Roles of four Arabidopsis U-box E3 ubiquitin ligases in negative regulation of abscisic acid-mediated drought stress responses. Plant Physiol. 2012;160(1):556-68.

18. Cho SK, Ryu MY, Song C, Kwak JM, Kim WT. Arabidopsis PUB22 and PUB23 are homologous U-box E3 ubiquitin ligases that play combinatory roles in response to drought stress. Plant Cell. 2008:20(7):1899-914.

19. Bergler J, Hoth S. Plant U-box armadillo repeat proteins AtPUB18 and AtPUB19 are involved in salt inhibition of germination in Arabidopsis. Plant Biol (Stuttgart). 2011;13(5):725-30

20. Hwang JH, Seo DH, Kang BG, Kwak JM, Kim WT. Suppression of Arabidopsis AtPUB30 resulted in increased tolerance to salt stress during germination. Plant Cell Rep. 2015:34(2):277-89.

21. Byun MY, Cui LH, Oh TK, Jung YJ, Lee A, Park KY, Kang BG, Kim WT. Homologous U-box E3 ubiquitin ligases OsPUB2 and OsPUB3 are involved in the positive regulation of low temperature stress response in Rice (Oryza sativa L.). Front Plant Sci. 2017;8:16.

22. Hur YJ, Yi YB, Lee JH, Chung YS, Jung HW, Yun DJ, Kim KM, Park DS, Kim $\mathrm{DH}$. Molecular cloning and characterization of OsUPS, a U-box containing E3 ligase gene that respond to phosphate starvation in rice (Oryza sativa). Mol Biol Rep. 2012;39(5):5883-8.

23. Salt JN, Yoshioka K, Moeder W, Goring DR. Altered germination and subcellular localization patterns for PUB44/SAUL1 in response to stress and phytohormone treatments. PLoS One. 2011;6(6):e21321.

24. Kinoshita A, ten Hove CA, Tabata R, Yamada M, Shimizu N, Ishida T, Yamaguchi K, Shigenobu S, Takebayashi Y, luchi S, et al. A plant U-box protein, PUB4, regulates asymmetric cell division and cell proliferation in the root meristem. Development. 2015:142(3):444-53.

25. Hu X, Qian Q, Xu T, Zhang Y, Dong G, Gao T, Xie Q, Xue Y. The U-box E3 ubiquitin ligase TUD1 functions with a heterotrimeric $\mathrm{G}$ alpha subunit to regulate Brassinosteroid-mediated growth in rice. PLoS Genet. 2013;9(3):e1003391.

26. Trujillo M, Ichimura K, Casais C, Shirasu K. Negative regulation of PAMPtriggered immunity by an E3 ubiquitin ligase triplet in Arabidopsis. Curr Biol. 2008;18(18):1396-401.

27. Lu D, Lin W, Gao X, Wu S, Cheng C, Avila J, Heese A, Devarenne TP, He P, Shan L. Direct ubiquitination of pattern recognition receptor FLS2 attenuates plant innate immunity. Science (New York). 2011;332(6036): 1439-42.

28. Zeng LR, Qu S, Bordeos A, Yang C, Baraoidan M, Yan H, Xie Q, Nahm BH, Leung H, Wang GL. Spotted leaf11, a negative regulator of plant cell death and defense, encodes a U-box/armadillo repeat protein endowed with E3 ubiquitin ligase activity. Plant Cell. 2004;16(10):2795-808.

29. Liu J, Park CH, He F, Nagano M, Wang M, Bellizzi M, Zhang K, Zeng X, Liu W, Ning Y, et al. The RhoGAP SPIN6 associates with SPL11 and OsRac1 and negatively regulates programmed cell death and innate immunity in rice. PLoS Pathog. 2015;11(2):e1004629.
30. Ishikawa K, Yamaguchi K, Sakamoto K, Yoshimura S, Inoue K, Tsuge S, Kojima C, Kawasaki T. Bacterial effector modulation of host E3 ligase activity suppresses PAMP-triggered immunity in rice. Nat Commun. 2014;5:5430.

31. Wang J, Qu B, Dou S, Li L, Yin D, Pang Z, Zhou Z, Tian M, Liu G, Xie Q, et al. The E3 ligase OsPUB15 interacts with the receptor-like kinase PID2 and regulates plant cell death and innate immunity. BMC Plant Biol. 2015;15:49.

32. Yang CW, Gonzalez-Lamothe R, Ewan RA, Rowland O, Yoshioka H, Shenton M, Ye H, O'Donnell E, Jones JD, Sadanandom A. The E3 ubiquitin ligase activity of arabidopsis PLANT U-BOX 17 and its functional tobacco homolog ACRE276 are required for cell death and defense. Plant Cell. 2006;18(4):1084-98.

33. Zhou J, Lu D, Xu G, Finlayson SA, He P, Shan L. The dominant negative ARM domain uncovers multiple functions of PUB13 in Arabidopsis immunity, flowering, and senescence. J Exp Bot. 2015;66(11):3353-66.

34. Zhang G, Zhang M, Zhao Z, Ren Y, Li Q, Wang W. Wheat TaPUB1 modulates plant drought stress resistance by improving antioxidant capability. Sci Rep. 2017;7(1):7549

35. Zhu Y, Li Y, Fei F, Wang Z, Wang W, Cao A, Liu Y, Han S, Xing L, Wang H, et al. E3 ubiquitin ligase gene CMPG1-V from Haynaldia villosa L. contributes to powdery mildew resistance in common wheat (Triticum aestivum L.). Plant J. 2015:84(1):154-68.

36. Mascher M, Gundlach H, Himmelbach A, Beier S, Twardziok SO, Wicker T, Radchuk V, Dockter C, Hedley PE, Russell J, et al. A chromosome conformation capture ordered sequence of the barley genome. Nature. 2017:544(7651):427-33.

37. Seo DH, Ahn MY, Park KY, Kim EY, Kim WT. The N-terminal UND motif of the Arabidopsis U-box E3 ligase PUB18 is critical for the negative regulation of ABA-mediated stomatal movement and determines its ubiquitination specificity for exocyst subunit Exo70B1. Plant Cell. 2016;28(12):2952-73.

38. Heise A, Lippok B, Kirsch C, Hahlbrock K. Two immediate-early pathogenresponsive members of the AtCMPG gene family in Arabidopsis thaliana and the W-box-containing elicitor-response element of AtCMPG1. Proc Natl Acad Sci U S A. 2002;99(13):9049-54.

39. Gonzalez-Lamothe R, Tsitsigiannis DI, Ludwig AA, Panicot M, Shirasu K Jones JD. The U-box protein CMPG1 is required for efficient activation of defense mechanisms triggered by multiple resistance genes in tobacco and tomato. Plant Cell. 2006;18(4):1067-83.

40. Kobayashi S, Tsugama D, Liu S, Takano T. A U-box E3 ubiquitin ligase, PUB20, interacts with the Arabidopsis G-protein beta subunit, AGB1. PLoS One. 2012;7(11):e49207.

41. Stegmann M, Anderson RG, Ichimura K, Pecenkova T, Reuter P, Zarsky V, McDowell JM, Shirasu K, Trujillo M. The ubiquitin ligase PUB22 targets a subunit of the exocyst complex required for PAMP-triggered responses in Arabidopsis. Plant Cell. 2012;24(11):4703-16.

42. Rajaraman J, Douchkov D, Luck S, Hensel G, Nowara D, Pogoda M, Rutten T, Meitzel T, Brassac J, Hofle C, et al. Evolutionarily conserved partial gene duplication in the Triticeae tribe of grasses confers pathogen resistance. Genome Biol. 2018;19(1):116.

43. Cho SK, Bae H, Ryu MY, Wook Yang S, Kim WT. PUB22 and PUB23 U-BOX E3 ligases directly ubiquitinate RPN6, a 26S proteasome lid subunit, for subsequent degradation in Arabidopsis thaliana. Biochem Biophys Res Commun. 2015:464(4):994-9.

44. Monaghan J, Xu F, Gao M, Zhao Q, Palma K, Long C, Chen S, Zhang Y, Li X. Two Prp19-like U-box proteins in the MOS4-associated complex play redundant roles in plant innate immunity. PLoS Pathog. 2009;5(7):e1000526.

45. Mayer KF, Waugh R, Brown JW, Schulman A, Langridge P, Platzer M, Fincher GB, Muehlbauer GJ, Sato K, Close TJ, et al. A physical, genetic and functional sequence assembly of the barley genome. Nature. 2012:491(7426):711-6.

46. Finn RD, Coggill P, Eberhardt RY, Eddy SR, Mistry J, Mitchell AL, Potter SC, Punta M, Qureshi M, Sangrador-Vegas A, et al. The Pfam protein families database: towards a more sustainable future. Nucleic Acids Res. 2016;44(D1):D279-85.

47. Finn RD, Attwood TK, Babbitt PC, Bateman A, Bork P, Bridge AJ, Chang HY, Dosztanyi Z, El-Gebali S, Fraser M, et al. InterPro in 2017-beyond protein family and domain annotations. Nucleic Acids Res. 2017;45(D1):D190-d199.

48. Matsumoto T, Tanaka T, Sakai H, Amano N, Kanamori H, Kurita K, Kikuta A, Kamiya K, Yamamoto M, Ikawa $\mathrm{H}$, et al. Comprehensive sequence analysis of 24,783 barley full-length cDNAs derived from 12 clone libraries. Plant Physiol. 2011;156(1):20-8

49. Kumar S, Stecher G, Tamura K. MEGA7: molecular evolutionary genetics analysis version 7.0 for bigger datasets. Mol Biol Evol. 2016;33(7):1870-4. 